\title{
Ceramic Fracture Following Cervical Disc Arthroplasty
}

\author{
A Case Report

\begin{abstract}
Ngoc Quyen Nguyen, MD, Dinesh Kafle, MD, Jacob M. Buchowski, MD, MS, Kun-Woo Park, MD, Bong-Soon Chang, MD, Choon-Ki Lee, MD, and Jin S. Yeom, MD
\end{abstract} \\ Investigation performed at the Department of Orthopaedic Surgery, Seoul National University, Seoul, Republic of Korea, \\ and the Department of Orthopaedic Surgery, Washington University in St. Louis, St. Louis, Missouri
}

A lthough ceramic fractures have been reported following total hip arthroplasty ${ }^{1-6}$, with an incidence ranging from $0.004 \%$ to $1.4 \%{ }^{1-3}$, no cases of ceramic fracture following cervical disc arthroplasty have been reported, to our knowledge. We present the case of a patient with cervical radiculopathy who underwent total disc replacement at C5-C6 and C6-C7, which was complicated by the development of recurrent symptoms approximately one month after the index procedure. At the time of the revision surgery, the ceramic insert at the C6-C7 level was found to be fractured on its convex cranial side. The implant used was a semiconstrained ceramicon-ceramic prosthesis (Discocerv Cervidisc Evolution; Scient'x, Guyancourt, France). Since the introduction of this prosthesis in April 2006 ${ }^{7}$, more than 2000 prostheses have been implanted. The patient was informed that data concerning the case would be submitted for publication, and he gave his consent.

\section{Case Report}

A fifty-five-year-old man presented with recurrent neck pain with radiation to the right upper extremity as well as right upper-extremity weakness and muscle atrophy. The pain was rated as 8 on a visual analogue scale (VAS) ranging from 0 to 10 . The radiating pain was most prominent in the posterolateral part of the arm and forearm and the dorsal part of the hand. The patient had no pain in the thumb or fingers. The Neck Disability Index (NDI) score ${ }^{8}$ was $36 \%$. Three months prior to presentation at our facility, the patient had undergone cervical disc arthroplasty at another institution with use of ceramic-onceramic prostheses at C5-C6 and C6-C7 for cervical spondylotic radiculopathy. This type of implant is a semiconstrained prosthesis composed of two parts, which allow spherical motion in a ball-and-socket joint design. It includes a spherical

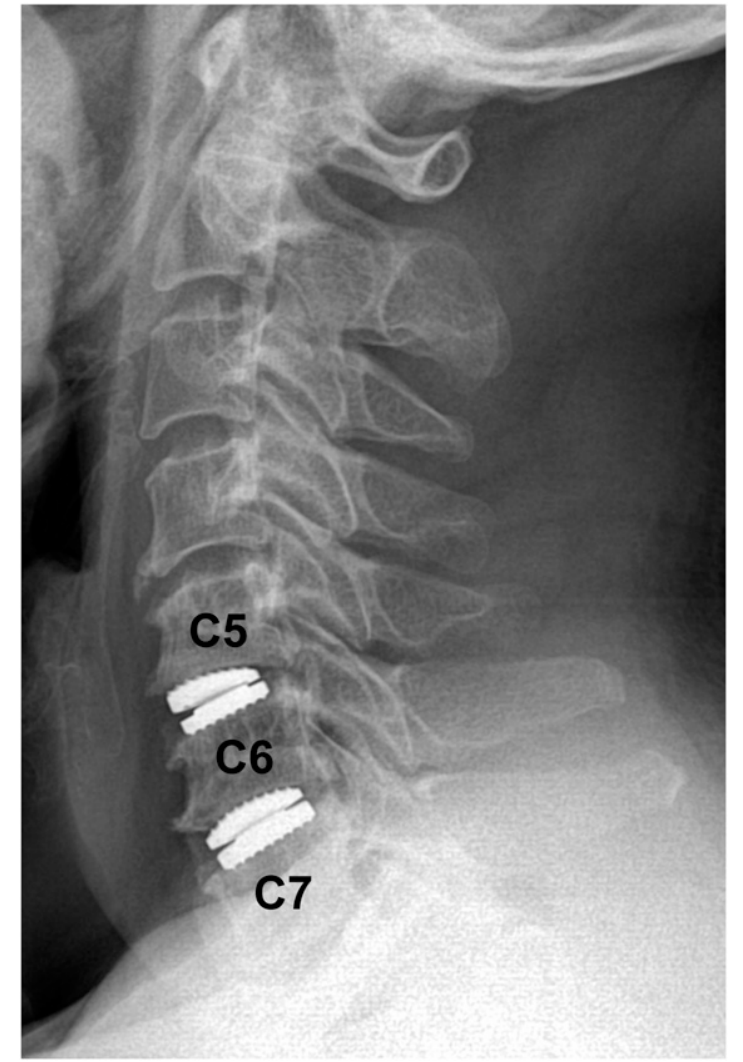

Fig. 1

Lateral cervical spine radiograph showing artificial discs at the C5-C6 and C6-C7 levels with no definite evidence of ceramic fracture, although, in retrospect, the distance between the metal plates at the $\mathrm{C6}-\mathrm{C} 7$ level appears slightly decreased compared with that at the C5-C6 level.

Disclosure: None of the authors received payments or services, either directly or indirectly (i.e., via his or her institution), from a third party in support of any aspect of this work. One or more of the authors, or his or her institution, has had a financial relationship, in the thirty-six months prior to submission of this work, with an entity in the biomedical arena that could be perceived to influence or have the potential to influence what is written in this work. No author has had any other relationships, or has engaged in any other activities, that could be perceived to influence or have the potential to influence what is written in this work. The complete Disclosures of Potential Conflicts of Interest submitted by authors are always provided with the online version of the article. 
The Journal of Bone \& Joint Surgery • JBjS. org Volume 93-A · Number 22 - November 16, 2011

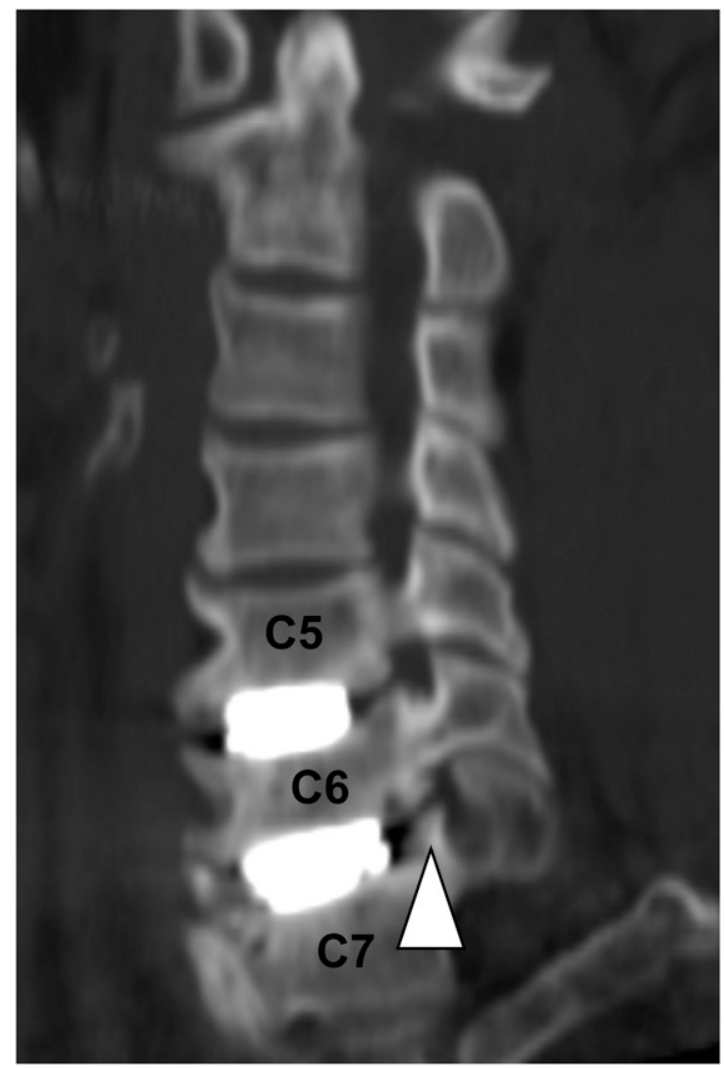

Fig. 2

CT image with sagittal foraminal oblique reconstruction showing a right uncoforaminal spur (arrowhead) at C6-C7.

convex head on the cranial insert and a spherical concave cup on the caudal insert. These two inserts are made of ceramic material: alumina for the cranial insert and zirconia for the caudal insert ${ }^{7}$. Each insert is backed with a metal plate. Before the initial operation, the patient had had similar symptoms; on the VAS scale, the pain in both the neck and the upper extremities was rated as 7 of 10 . Following the initial surgery, he had had substantial improvement in symptoms. Approximately one month later, he began to develop recurrent symptoms. The onset of the recurrent pain was relatively gradual rather than sudden. He denied having any notable history of trauma, sports activity such as running, or a crunching or cracking sensation or sound in the neck between the first operation and the recurrence of symptoms. He also did not have a squeaking sound or sensation in the neck.

On evaluation, the patient had muscle atrophy of the posterolateral part of the right upper arm and the radial part of the right forearm. He reported that the muscle atrophy had been present for at least six months prior to the index procedure and had not changed following the initial operation. Motor strength in the right elbow extensor, wrist extensor, and finger flexor muscles was 5-/5. Radiographs demonstrated seemingly intact prostheses at C5-C6 and C6-C7 (Fig. 1). No instability was observed on flexion-extension radiographs. A magnetic resonance imaging (MRI) scan and a computed
Ceramic Fracture Following Cervical

Disc Arthroplasty

tomography (CT) scan (Fig. 2) demonstrated a large right paracentral spur at C5-C6 and foraminal stenosis at C6-C7 on the right, raising the question as to whether he should have undergone cervical disc arthroplasty initially. Since he had had no improvement in symptoms with nonoperative treatment, the patient requested revision surgery. Given the persistent spondylotic changes and inadequate decompression at both levels as well as evidence of $\mathrm{C} 6$ and $\mathrm{C} 7$ radiculopathy, removal of the cervical arthroplasty devices and anterior cervical fusion from $\mathrm{C} 5$ to $\mathrm{C} 7$ was recommended.

Intraoperatively, the metal plates of the implants were well fixed to the vertebral end plates; they were separated from the end plates without difficulty by gently inserting a $5-\mathrm{mm}$ wide osteotome between the metal plate and the end plate with use of a mallet. There was no notable bone loss during implant removal. After removal of the cranial metal plate from the C6$\mathrm{C} 7$ level, we noted that the ceramic ball in the cranial part of the implant had been comminuted into approximately ten pieces (Fig. 3), while the portion of the ceramic insert within the cranial metal plate remained unfractured (Fig. 4). The fractured fragments were surrounded by scar tissue around the metal plates and thus did not cause direct compression on or injury to the neurovascular tissues with their sharp edges. There were numerous scratches in the anterior parts of both the cranial and the caudal metal plate (Fig. 4). A complete foraminotomy on the right side and an anterior cervical fusion were performed (Fig. 5). At C5-C6, the implant was intact.

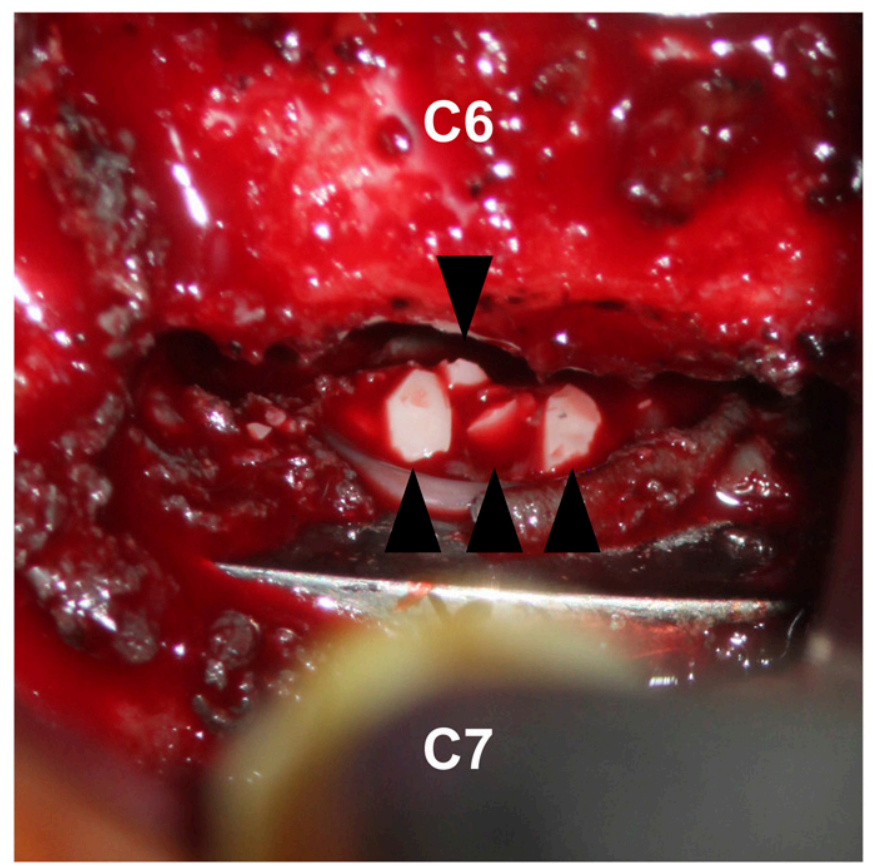

Fig. 3

Intraoperative photograph of the C6-C7 level, made after removal of the cranial part of the artificial disc from the lower end plate of $\mathrm{C6}$, demonstrating four fractured fragments (arrows) of the ceramic ball lying on the remaining caudal part of the implant. The other fragments have already been removed. 
The Journal of Bone \& Joint Surgery $\cdot$ JBjS.org Volume 93-A • Number 22 - November 16, 2011

Ceramic Fracture Following Cervical

Disc Arthroplasty
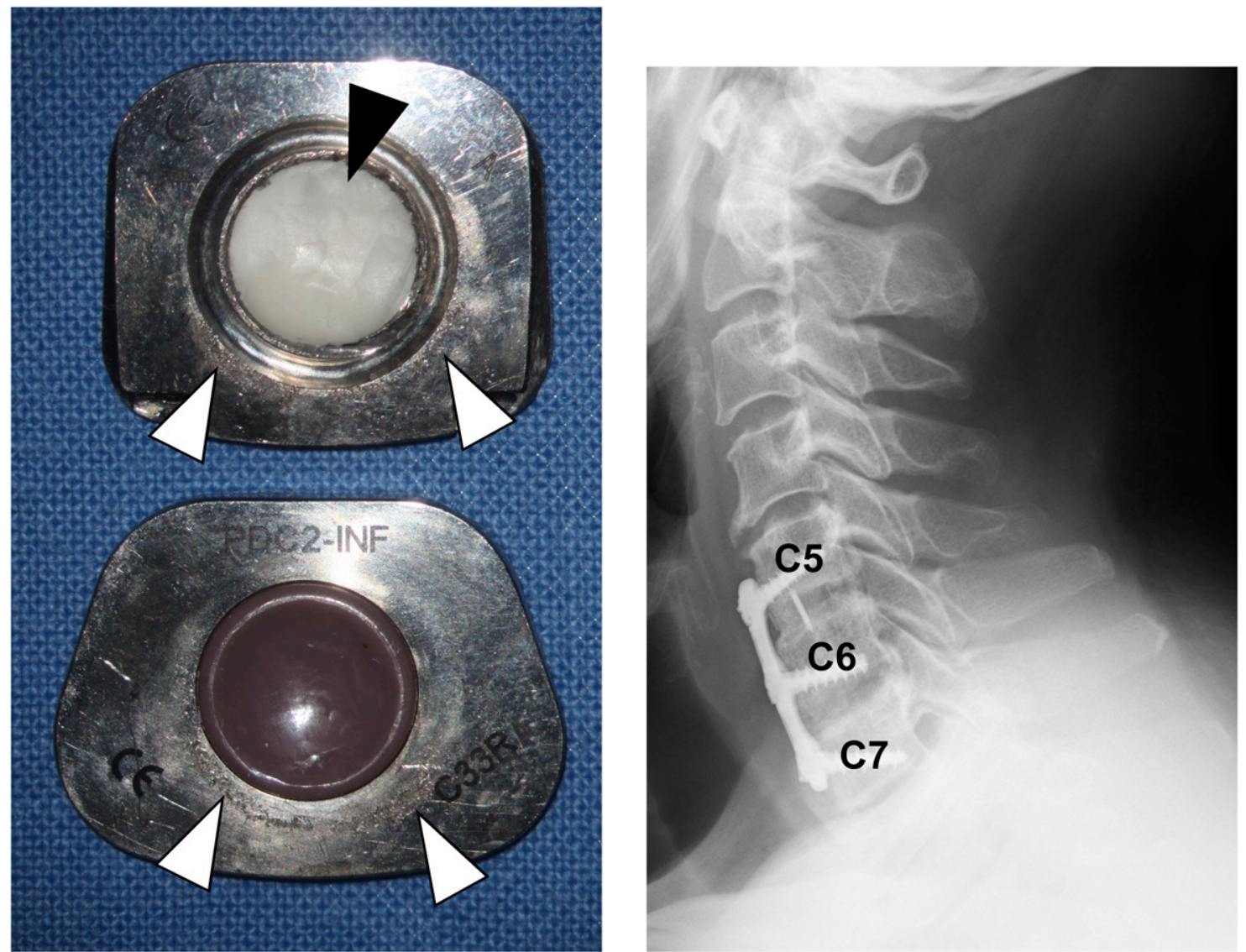

Fig. 4

Fig. 5

Fig. 4 Photograph of the implant removed from the $\mathrm{C} 6-\mathrm{C} 7$ level. The part of the ceramic insert located within the cranial metal plate (black arrowhead) remains intact, although the ceramic ball had been fractured off and is not seen in this photograph. The anterior parts of both the cranial and the caudal metal plate are scratched (white arrowheads). These scratches were likely made by the sharp edges of the fractured ceramic fragments. Fig. $\mathbf{5}$ Lateral cervical spine radiograph obtained six months following the revision operation, demonstrating anterior cervical fusion and plate fixation from $\mathrm{C} 5$ to $\mathrm{C} 7$.

After implant removal, resection of the paracentral spurs and the anterior cervical fusion were performed from $\mathrm{C} 5$ to $\mathrm{C} 7$. The pain was greatly alleviated immediately after the surgery, and at the time of follow-up at six months after this revision both the neck pain and the arm pain were rated as 1 of 10 on the VAS scale.

\section{Discussion}

$\mathrm{O}$ ur patient denied experiencing trauma or participating in sports activity that might have caused notable axial impact loading. We believe that some degree of repetitive shear force is likely to be applied on the ceramic ball of the artificial disc even without trauma, since there is normal anteroposterior motion coupled with flexion and extension. The fact that the part of the ceramic insert within the cranial metal plate remained intact while the convex-ball portion was fractured supports the hypothesis that shear force was the cause of fracture. Had it been axial impact loading, one might expect that both parts of the cranial ceramic insert would have been fractured. Since this semiconstrained implant permits only spherical motion and does not allow anteroposterior motion, shear forces to the convex portion of the ceramic implant could have been present, predisposing the ceramic implant to fracture, especially since ceramics are known to be brittle with shear forces ${ }^{1}$.

Surprisingly, fracture of the ceramic component was not diagnosed prior to the second surgical procedure. Prior to that surgery, we assumed that the disc height elevation along with partial decompression achieved during the index operation alleviated the patient's symptoms transiently but that this effect did not last long as the foraminal height decreased with slow settling of the prosthesis into the end plates. Since a wide decompression is needed when disc arthroplasty is performed and the decompression in our case appeared inadequate, the patient could have had recurrence of symptoms even without device failure, as inadequate decompression is reported to be the leading cause of arthroplasty failure'. Nevertheless, we believe that ceramic fracture with consequent disc and foraminal height reduction led to or contributed to the recurrence of the symptoms. The effect of the fractured ceramic pieces also could have contributed, although the pieces were surrounded by scar tissue and did not appear to cause direct neural injury or compression. 
The Journal of Bone \& Joint Surgery · Jbjs. org Volume 93-A · Number 22 - November 16, 2011
Ceramic Fracture Following Cervical

Disc Arthroplasty
An important consideration in ceramic fracture following cervical disc arthroplasty is whether the implant fracture can be diagnosed without surgical exploration. Since our patient did not have a history of abnormal impact loading, did not feel a crunching, cracking, or squeaking sensation or sound, and had no acute onset of symptoms, there were no clinical reasons to suspect fracture of the prosthesis. Most alarmingly, the imaging studies yielded no clues to suggest that the ceramic component had fractured. Even in retrospect, with full knowledge of the events, the ceramic fracture was not seen on the imaging studies. Evaluation of the prosthesis on the CT scan (Fig. 2) and MRI was limited by the metal artifact. Even though the distance between the metal plates at C6-C7 on the lateral radiograph may appear slightly decreased, the decrease is minimal and was considered an indeterminate finding (Fig. 1), possibly because the fragments were contained in the concavity of the lower liner, preventing total collapse.

Although this report is limited by the lack of explant analysis and histological evaluation, several lessons can be learned from this case. First and foremost, spine surgeons need to be aware that ceramic components can fracture following cervical disc arthroplasty. These fractures can be difficult to diagnose since patients may not have symptoms suggestive of a fracture and a fracture may not be appreciated or visible on imaging studies. A ceramic fracture should, therefore, be suspected when a patient presents with unexplained neck pain and/or radiating pain after cervical disc arthroplasty with a ceramic prosthesis. Finally, we believe that when a fractured component is found intraoperatively, the prosthesis should be analyzed to determine the mode of failure and histological analysis should be performed.

Note: The authors thank Yoon Ju Kwon and Seung Min You, the spine research coordinators at our institution, for their contributions to the project.

Ngoc Quyen Nguyen, MD

Dinesh Kafle, MD

Kun-Woo Park, MD

Jin S. Yeom, MD

Spine Center and Department of Orthopaedic Surgery,

Seoul National University College of Medicine

and Seoul National University Bundang Hospital,

166 Gumiro, Bundang-ku, Sungnam,

463-707, Republic of Korea.

E-mail address for J.S. Yeom: highcervical@gmail.com

Jacob M. Buchowski, MD, MS

Department of Orthopaedic Surgery,

Washington University in St. Louis,

660 South Euclid Avenue, Campus Box 8233,

St. Louis, MO 63110

Bong-Soon Chang, MD

Choon-Ki Lee, MD

Department of Orthopaedic Surgery,

Seoul National University Hospital,

Seoul National University School of Medicine,

101 Daehangno, Jongno-gu, Seoul, 110-744, Republic of Korea

\section{References}

1. Hannouche D, Nich C, Bizot P, Meunier A, Nizard R, Sedel L. Fractures of ceramic bearings: history and present status. Clin Orthop Relat Res. 2003;417:19-26.

2. Willmann G. Ceramic femoral head retrieval data. Clin Orthop Relat Res. 2000; 379:22-8.

3. Koo KH, Ha YC, Jung WH, Kim SR, Yoo JJ, Kim HJ. Isolated fracture of the ceramic head after third-generation alumina-on-alumina total hip arthroplasty. J Bone Joint Surg Am. 2008;90:329-36.

4. Sariali E, Stewart T, Mamoudy P, Jin Z, Fisher J. Undetected fracture of alumina ceramic on ceramic hip prosthesis. J Arthroplasty. 2010;25:658.e1-5. Epub 2009 Jul 4.

5. Torán MM, Cuenca J, Martinez AA, Herrera A, Thomas JV. Fracture of the ceramic femoral head after ceramic-on-ceramic total hip arthroplasty. J Arthroplasty. 2006; 21:1072-3.
6. Rhoads DP, Baker KC, Israel R, Greene PW. Fracture of an alumina femoral head used in ceramic-on-ceramic total hip arthroplasty. J Arthroplasty. 2008;23:1239. e25-30. Epub 2008 Apr 11.

7. Ramadan AS, Mitulescu A, Schmitt P. Total cervical disc replacement with the Discocerv ${ }^{\circledR}$ (Cervidisc Evolution) cervical prosthesis: early results of a second generation. Eur J Orthop Surg Traumatol. 2007;17:513-20.

8. Vernon H, Mior S. The Neck Disability Index: a study of reliability and validity. J Manipulative Physiol Ther. 1991;14:409-15.

9. Anderson PA, Rouleau JP, Toth JM, Riew KD. A comparison of simulator-tested and -retrieved cervical disc prostheses. Invited submission from the Joint Section Meeting on Disorders of the Spine and Peripheral Nerves, March 2004. J Neurosurg Spine. 2004;1:202-10. 